\title{
Erratum to: Local Interleukin-18 System in the Basolateral Amygdala Regulates Susceptibility to Chronic Stress
}

\author{
Tae-Kyung Kim ${ }^{1}$ • Ji-Eun Kim ${ }^{1}$ • Juli Choi ${ }^{1}$. Jin-Young Park ${ }^{1}$ • Jung-Eun Lee ${ }^{1}$. \\ Eun-Hwa Lee ${ }^{1} \cdot$ Yunjin Lee $^{1} \cdot$ Bo Yeon Kim ${ }^{2}$ - Young $\mathrm{J} \mathrm{Oh}^{3} \cdot$ Pyung-Lim Han ${ }^{1,4}$
}

Published online: 7 October 2016

(C) Springer Science+Business Media New York 2016

\section{Erratum to: Mol Neurobiol \\ DOI 10.1007/s12035-016-0052-7}

The article unfortunately have serious errors in Figures 2 and 5 captions. With these, the authors hereby publish the correct captions.

Fig. 2 Expression of IL18 and IL18 receptors in the BLA. a Photomicrograph showing the location (red rectangle) of high magnification images $(\mathbf{b}, \mathbf{c}, \mathbf{f}, \mathbf{g})$ and the approximate area in the BLA used for quantifications (blue circle). CeA central amygdala, BLA basolateral amygdala. Scale bar $=200 \mu \mathrm{m}$. b-e Photomicrographs showing the co-staining of proIL18 and GAD67 (b), and proIL18 and Glu4 (c) in the BLA of mice treated with $2 \mathrm{~h} \times 14 \mathrm{~d}$ RST. Venn diagrams for the quantification of co-localization of proIL18 and GAD67 (d) and proIL18 and Glu4 (e), and the number of counted cells of each marker. f-i Photomicrographs showing the co-staining of

The online version of the original article can be found at http://dx.doi. org/10.1007/s12035-016-0052-7.

Young J. Oh

yjoh@yonsei.ac.kr

Pyung-Lim Han

plhan@ewha.ac.kr

1 Department of Brain and Cognitive Sciences, Ewha Womans University, Seoul 120-750, Republic of Korea

2 World Class Institute, Korea Research Institute of Bioscience \& Biotechnology, Science Park, Cheongwon 363-883, South Korea

3 Department of Systems Biology, Yonsei University College of Life Science and Biotechnology, Seoul 120-749, South Korea

4 Chemistry and Nano Science, Ewha Womans University, Seoul, Republic of Korea
IL18R $\alpha$ and GAD67 (f), and IL18R $\alpha$ and Glu4 (g) in the BLA of mice treated with $2 \mathrm{~h} \times 14 \mathrm{~d}$ RST. Venn diagrams for the quantification of co-localization of IL $18 \mathrm{R} \alpha$ and GAD67 (h) and IL18R $\alpha$ and Glu4 (i), and the number of counted cells of each marker. Scale bars in $(\mathbf{c}, \mathbf{g})=20 \mu \mathrm{m}$.

Fig. 5 NF-kB KO mice were resilient to chronic stress that promotes depression-like behaviors. a Experimental design for treatment with $2 \mathrm{~h} \times 14 \mathrm{~d}$ RST and subsequent behavioral tests. Behavioral tests were performed in the order of the sociability test, TST, FST, and NSF. b-e Social interaction levels in the U-field assay (b), immobility time in the TST (c) and FST (d), and latency to eating in the NSF test (e) of NF-kB KO mice and WTcontrols. $n=7-9$. f Experimental design for foot-shock treatment for $1 \mathrm{~h}$ daily for 7 days and subsequent behavioral tests. Behavioral tests were performed on post-stress days 1-3 in the sequence of the sociability test, TST, and FST. The sucrose preference test was performed independently from the other tests. $\mathbf{g}-\mathbf{k}$ Sucrose intake in the sucrose preference test (g), social interactions in the U-field assay (h), immobility times in the TST (i) and FST (j), and latency to eating in the NSF test (k) among NF-kB KO mice and WT controls. $n=8-12$. I Realtime PCR data showing the downregulation of Hcrt, $\mathrm{MCH}, \mathrm{OXT}$, AVP, and TRH in the amygdala of NF-kB p50 $\mathrm{KO}$ (NF-kB KO) mice. The downregulation of Hcrt, $\mathrm{MCH}$, and OXT were significant. $n=6$ animals for each, four repeats of PCR. m Real-time PCR data showing the expression levels of G9a in the amygdala 3 days after siRNA injection, of naïve normal mice injected with siRNA-control (CON-siCON) or mice subjected to $2 \mathrm{~h} \times 14 \mathrm{~d}$ RST, followed by injection of siRNA-control (RST-siCON), siRNA-IL18 (RST-silL18), siRNA-IL18R $\alpha$ (RSTT-siIL18Ra), siRNA-NF-kB (RSTsiNF-kB), or siRNA-STAT3 (RST-siSTAT3). $n=6$ animals for each, four repeats of PCR. $\mathbf{n}$ Real-time PCR data showing the expression levels of G9a in the amygdala of IL18 KO mice 
(IL18 KO) or NF-kB p50 KO mice (NF-kB KO). $n=6$ animals for each, four repeats of PCR. Data are presented as mean \pm SEM. $*$ and $* *$ denote the difference between indicated groups (b-e, g-k) or from WTcontrol (l) and CON-siCON (m) at $p<0.05$ and $p<0.01$, respectively. \# and \#\# denote the difference from RST-siCON (m) at $p<0.05$ and $p<0.01$, respectively (Student's t test, one-way or two-way ANOVA and Newman-Keuls post hoc test). 